\title{
Mixed Fractional Heat Equation Driven by Fractional Brownian Sheet and Lévy Process
}

\author{
Dengfeng Xia, ${ }^{1,2}$ Litan Yan, ${ }^{1,3}$ and Weiyin Fei $^{2}$ \\ ${ }^{1}$ College of Information Science and Technology, Donghua University, 2999 North Renmin Rd., Songjiang, \\ Shanghai 201620, China \\ ${ }^{2}$ School of Mathematics and Physics, Anhui Polytechnic University, Wuhu, Anhui 241000, China \\ ${ }^{3}$ Department of Mathematics, College of Science, Donghua University, 2999 North Renmin Rd., Songjiang, \\ Shanghai 201620, China \\ Correspondence should be addressed to Litan Yan; litan-yan@hotmail.com
}

Received 23 December 2016; Revised 20 March 2017; Accepted 21 March 2017; Published 13 April 2017

Academic Editor: Maria L. Gandarias

Copyright (C) 2017 Dengfeng Xia et al. This is an open access article distributed under the Creative Commons Attribution License, which permits unrestricted use, distribution, and reproduction in any medium, provided the original work is properly cited.

We consider the stochastic heat equation of the form $\partial u / \partial t=\left(\Delta+\Delta_{\alpha}\right) u+(\partial f / \partial x)(t, x, u)+\sigma(t, x, u) \dot{L}+\dot{W}^{H}$, where $\dot{W}^{H}$ is the fractional noise, $\dot{L}$ is a (pure jump) Lévy space-time white noise, $\Delta$ is Laplacian, and $\Delta_{\alpha}=-(-\Delta)^{\alpha / 2}$ is the fractional Laplacian generator on $\mathbb{R}$, and $f, \sigma:[0, T] \times \mathbb{R} \times \mathbb{R} \rightarrow \mathbb{R}$ are measurable functions. We introduce the existence and uniqueness of the solution by the fixed point principle under some suitable assumptions.

\section{Introduction}

Stochastic calculus of fractional Brownian motion (in short, $\mathrm{fBm}$ ) naturally led to the study of stochastic partial differential equations (in short, SPDEs) driven by it, and the study of such SPDEs constitutes an important research direction in probability theory and stochastic analysis, and many interesting researches have been done. The motivation comes from wide applications of $\mathrm{fBm}$. We refer, among others, to Duncan et al. [1], Hu [2], Jiang et al. [3, 4], Liu and Yan [5], Sobczyk [6], Tindel et al. [7], Mishura et al. [8], and the references therein. On the other hand, as is well known, SPDEs driven by Lévy noise constitute a very important research direction and many significant researches have been carried out. We mention the works of Bo et al. $[9,10]$, Shi and Wang [11], Mueller [12], Chen et al. [13], Løkka et al. [14], and Truman and $\mathrm{Wu}[15,16]$. However, it is not sufficient to study the mixed heat equation with fractional and Lévy noises.

It is important to note that the increasing interest to study the pseudo-differential operators $\Delta_{\alpha}+\Delta_{\beta}$ is motivated by its applications to fluid dynamic traffic model, statistical mechanics, and heat conduction in materials with memory and also because they can be employed to approach nonlinear conservation laws. Therefore, it seems interesting to handle the mixed fractional heat equation. In the recent paper of Xia and Yan [17], they introduced only the existence and uniqueness of the solution of a mixed fractional heat equation driven by a fractional Brownian sheet. As an extension, in the present paper, we consider the stochastic heat equation of the form

$$
\begin{aligned}
\frac{\partial u(t, x)}{\partial t}= & \left(\Delta+\Delta_{\alpha}\right) u(t, x)+\frac{\partial f}{\partial x}(t, x, u(t, x)) \\
& +\sigma(t, x, u(t, x)) \dot{L}+\dot{W}^{H}, \\
u(0, x)= & u_{0}(x), \quad x \in \mathbb{R}, \quad x \in \mathbb{R},
\end{aligned}
$$

where $\Delta$ is Laplacian, $\Delta_{\alpha}=-(-\Delta)^{\alpha / 2}$ is the fractional Laplacian generator on $\mathbb{R}, \dot{W}^{H}$ is the fractional noise, and $\dot{L}$ is a (pure jump) Lévy space-time white noise. We first state two assumptions. 
Assumption 1. For each $T>0$, there exists a constant $C>0$ such that

$$
\begin{aligned}
& |f(t, x, y)| \leq C(1+|y|) \\
& |f(t, x, u)-f(t, x, \bar{u})|+|\sigma(t, x, u)-\sigma(t, x, \bar{u})| \\
& \quad \leq C|u-\bar{u}|
\end{aligned}
$$

for all $(t, x, y) \in[0, T] \times \mathbb{R} \times \mathbb{R}$ and $u, \bar{u} \in \mathbb{R}$.

Assumption 2. For some $p \geq 2$, we have

$$
\mathbb{E}\left[\left\|u_{0}(\cdot)\right\|_{p}^{p}\right]<\infty
$$

The structure of this paper is as follows. In Section 2, we briefly present some basic notations and preliminaries on the pseudo-differential operator $\Delta+\Delta_{\alpha}$, Lévy space-time white noise, and fractional noise. In Section 3, we study the existence and uniqueness of the Walsh-mild solution to (1).

\section{Preliminaries}

In this section, we briefly recall some basic results for Green function of the pseudo-differential operator $\Delta+\Delta_{\alpha}$ and stochastic calculus associated with fractional Brownian sheet and Lévy space-time white noise. We refer to Chen et al. $[18,19]$, Shi and Wang [11], Nualart [20], and the references therein for more details. In this paper, the letter $C$, with or without subscripts, stands for a positive constant whose value is unimportant and which may change from location to location, even within a line; we also stress that it depends on some constants.

2.1. Pseudo-Differential Operator $\Delta+\Delta_{\alpha}$. Consider a symmetric $\alpha$-stable motion $X^{\alpha}=\left\{X_{t}^{\alpha}, t \geq 0\right\}$ with $\alpha \in(0,2)$ and an independent standard Brownian motion $B$ on $\mathbb{R}^{d}$. Then, the process

$$
Y_{t}:=B_{t}+X_{t}^{\alpha}, \quad t \geq 0
$$

is a diffusion such that its transition density function $G^{(\alpha)}(x, t)$ satisfies

$$
\int_{\mathbb{R}^{d}} G^{(\alpha)}(x, t) e^{i z x} d x=e^{-t\left(|z|^{\alpha}+z^{2}\right)}
$$

for all $t \geq 0$ and $z \in \mathbb{R}^{d}$, and moreover $G^{(\alpha)}(x, t)$ is the fundamental solution of equation

$$
\frac{\partial u}{\partial t}=\left(\Delta+\Delta_{\alpha}\right) u
$$

The transition density function $G^{(\alpha)}$ is also called the heat kernel of the operator $\Delta+\Delta_{\alpha}$. Denote

$$
G^{(\alpha)}(s, y ; t, x)=G^{(\alpha)}(t-s, x-y)
$$

for all $x, y \in \mathbb{R}^{d}$ and $s, t \geq 0$. For the heat kernel $G^{(\alpha)}$, we have the following estimates (see, for examples, Chen et al. [18], Kolokoltsov [21], and Bass and Levin [22]):

$$
\begin{aligned}
& G^{(\alpha)}(s, y ; t, x)=G^{(\alpha)}(t-s, x-y) \\
& \leq C_{1}\left((t-s)^{-d / 2} e^{-|x-y|^{2} / C_{2}(t-s)}+(t-s)^{-d / 2}\right. \\
& \left.\wedge \frac{(t-s)}{|x-y|^{d+\alpha}}\right), \\
& \left|\frac{\partial G^{(\alpha)}}{\partial t}(s, y ; t, x)\right| \leq \frac{C}{|t-s|} G^{(\alpha)}(s, y ; t, x), \\
& \left|\frac{\partial G^{(\alpha)}}{\partial y}(s, y ; t, x)\right| \leq C\left(|t-s|^{-1 / \alpha} \wedge \frac{1}{|x-y|}\right) \\
& \cdot G^{(\alpha)}(s, y ; t, x)
\end{aligned}
$$

for all $t>s>0, x, y \in \mathbb{R}^{d}$ and some constants $C, C_{1}, C_{2}>$ 1 , where $a_{1} \wedge a_{2}:=\min \left\{a_{1}, a_{2}\right\}$ for $a_{1}, a_{2} \in \mathbb{R}$. In this paper, we only consider the case $d=1$.

2.2. Lévy Space-Time White Noise. Let $(\Omega, \mathscr{F}, P)$ be a complete probability space with a usual filtration $\left\{\mathscr{F}_{t}\right\}_{t \geq 0}$ and let $\left(U_{i}, \mathscr{B}\left(U_{i}\right)\right)(i=1,2)$ be two arbitrary measure spaces; $v_{i}$ is a $\sigma$-finite measure defined on $\left(U_{i}, \mathscr{B}\left(U_{i}\right)\right)$ for $i=1,2$. Following, for example, Ikeda and Watanabe [23] or Truman and $\mathrm{Wu}[16]$, we denote

$$
\begin{aligned}
N & :\left(U_{1}, \mathscr{B}\left(U_{1}\right), v_{1}\right) \times\left(U_{2}, \mathscr{B}\left(U_{2}\right), v_{2}\right) \times(\Omega, \mathscr{F}, P) \\
& \longrightarrow \mathbb{N} \cup\{\infty\},
\end{aligned}
$$

which is called a Poisson random measure on $\left(U_{1}, \mathscr{B}\left(U_{1}\right), v_{1}\right)$, if, for all $A_{1} \in \mathscr{B}\left(U_{1}\right), A_{2} \in \mathscr{B}\left(U_{2}\right)$ and $n \in \mathbb{N} \cup\{\infty\}$,

$$
\begin{aligned}
P( & \left.\omega \in \Omega: N\left(A_{1}, A_{2}, \omega\right)=n\right) \\
& =\frac{1}{n !} v_{1}^{n}\left(A_{1}\right) v_{2}^{n}\left(A_{2}\right) e^{-v_{1}\left(A_{1}\right) v_{2}\left(A_{2}\right)},
\end{aligned}
$$

where $\mathbb{N}=\{0,1,2, \ldots\}$.

In particular, when $U_{1}=[0, \infty) \times \mathbb{R}$, we define the compensating $\left\{\mathscr{F}_{t}\right\}$-martingale measure

$$
\begin{aligned}
Q\left(t, A_{1}, A_{2}\right):= & N\left([0, t] \times A_{1}, A_{2}\right) \\
& -t \nu_{1}\left(A_{1}\right) \nu_{2}\left(A_{2}\right),
\end{aligned}
$$

for all $\left(t, A_{1}, A_{2}\right) \in[0, \infty) \times \mathscr{B}(\mathbb{R}) \times \mathscr{B}\left(U_{2}\right)$ with $v_{1}([0, t] \times$ $\left.A_{1}\right) v_{2}\left(A_{2}\right)<\infty$.

For any $\left(\mathscr{F}_{t}\right)$-predictable integrand $\varphi:[0, \infty) \times \mathbb{R} \times U_{2} \times$ $\Omega \rightarrow \mathbb{R}$ which satisfies

$$
\mathbb{E} \int_{0}^{t} \int_{A_{1} \times A_{2}}|\varphi(s, x, y)|^{2} d s d x v_{2}(d y)<\infty,
$$

$$
\forall t \in[0, \infty)
$$


for some $\left(A_{1}, A_{2}\right) \in \mathscr{B}(\mathbb{R}) \times \mathscr{B}\left(U_{2}\right)$, we can define the stochastic integral

$$
\begin{aligned}
\int_{0}^{t+} & \int_{A_{1} \times A_{2}} \varphi(s, x, y) Q(d y, d x, d s) \\
:= & \int_{0}^{t+} \int_{A_{1} \times A_{2}} \varphi(s, x, y) N(d y, d x, d s) \\
& \quad-\int_{0}^{t} \int_{A_{1} \times A_{2}} \varphi(s, x, y) v_{2}(d y) d x d s,
\end{aligned}
$$

which is a square integrable $\left(\mathscr{F}_{t}\right)_{t \geq 0}$-martingale with the quadratic variation process

$$
\begin{gathered}
\left\langle\int_{0}^{+} \int_{A_{1} \times A_{2}} \varphi(s, x, y) Q(d y, d x, d s)\right\rangle_{t} \\
\quad=\int_{0}^{t} \int_{A_{1} \times A_{2}}|\varphi(s, x, y)|^{2} \nu_{2}(d y) d x d s .
\end{gathered}
$$

For the Poisson random measure $N$ and its compensating martingale measure $Q$, we can define the Radon-Nikodym derivatives

$$
\begin{aligned}
& \widetilde{N}(d y, x, t):=\frac{N(d t d x, d y)}{d t d x}, \\
& \widetilde{Q}(d y, x, t):=\frac{Q(d t d x, d y)}{d t d x}
\end{aligned}
$$

for $(t, x, y) \in[0, \infty) \times \mathbb{R} \times U_{2}$. A pure jump Lévy space-time white noise has the following structure:

$$
\begin{aligned}
\dot{L}(x, t)= & \int_{A_{0}} g_{1}(t, x, y) \widetilde{Q}(d y, x, t) \\
& +\int_{U_{2} \backslash A_{0}} g_{2}(t, x, y) \widetilde{N}(d y, x, t)
\end{aligned}
$$

for some $A_{0} \in U_{2}$ such that $v_{2}\left(U_{2} \backslash A_{0}\right)<\infty$ and $\int_{A_{0}} z^{2} v_{2}(d z)<+\infty$, where $g_{1}, g_{2}:[0, \infty) \times \mathbb{R} \times U_{2} \rightarrow \mathbb{R}$ are some measurable functions.

Next we quote the following B-D-G inequality (see, for example, [24] or [10]).

Proposition 3. Let $\varphi:[0, \infty) \times \mathbb{R} \times U_{2} \times \Omega \rightarrow \mathbb{R}$ be $\left(\mathscr{F}_{t}\right)_{t \geq 0^{-}}$ predictable and satisfy (15). Denote by $Y$ the integral process

$$
\left\{Y_{t}:=\int_{0}^{t+} \int_{\mathbb{R} \times U_{2}} \varphi(s, y, z) Q(d z, d y, d s), t \geq 0\right\} ;
$$

then for any $T>0$ and $p>1$, there exists a constant $C_{p, T}>0$ such that

$$
\begin{aligned}
& \sup _{t \in[0, T]} \mathbb{E}\left[\left|Y_{t}\right|^{p}\right] \\
& \leq C_{p, T}\left(\int_{0}^{T} \int_{\mathbb{R} \times U_{2}}\left(\mathbb{E}\left[|\varphi(s, y, z)|^{p}\right]\right)^{2 / p}\right. \\
& \left.\cdot v_{2}(d z) d y d s\right)^{p / 2} .
\end{aligned}
$$

In order to handle (1) we claim also the following assumptions.

Assumption 4. For $p \geq 2$, the mappings

$$
\begin{aligned}
\psi(t, x)= & \int_{U_{2} \backslash U_{0}} g_{2}(t, x, y) v_{2}(d y), \\
g(t, x, y)= & g_{1}(t, x, y) \mathbb{1}_{A_{0}}(y) \\
& +g_{2}(t, x, y) \mathbb{1}_{U_{2} \backslash A_{0}}(y)
\end{aligned}
$$

satisfy, respectively,

$$
\begin{array}{r}
\sup _{t \in[0, T]}\|\psi(t, \cdot)\|_{p}^{p}<\infty, \\
\sup _{t \in[0, T]}\left\|\int_{U_{2}}|g(t, \cdot, y)|^{2} \nu_{2}(d y)\right\|_{p / 2}^{p / 2}<\infty .
\end{array}
$$

2.3. Fractional Noises. Let $\mathscr{B}_{b}(\mathbb{R})$ denote a class of bounded Borel sets in $\mathbb{R}$ and $H \in(1 / 2,1)$. Assume that

$$
W^{H}=\left\{W^{H}\left([0, t] \times A_{1}\right), t \in[0, T], A_{1} \in \mathscr{B}_{b}(\mathbb{R})\right\}
$$

is a centered Gaussian family of random variables with the covariance

$$
\begin{aligned}
& \mathbb{E}\left(W^{H}\left([0, s] \times A_{1}\right) W^{H}\left([0, t] \times A_{2}\right)\right) \\
& \quad=\left|A_{1} \cap A_{2}\right| R_{H}(s, t), \\
& R_{H}(s, t)=\frac{1}{2}\left(|t|^{2 H}+|s|^{2 H}-|t-s|^{2 H}\right)
\end{aligned}
$$

for $s, t \in[0, T], A_{1}, A_{2} \in \mathscr{B}_{b}(\mathbb{R})$, where $\left|A_{1}\right|$ denotes the Lebesgue measure of the set $A_{1} \in \mathscr{B}_{b}(\mathbb{R})$.

Let $\mathcal{S}$ be the set of step functions on $[0, T] \times \mathbb{R}$ and let $\mathscr{H}$ be the Hilbert space defined as the closure of $\mathcal{S}$ with respect to the scalar product

$$
\left\langle\mathbb{1}_{[0, t] \times A_{1}}, \mathbb{1}_{[0, s] \times A_{2}}\right\rangle_{\mathscr{H}}=\left|A_{1} \cap A_{2}\right| R_{H}(t, s) .
$$

Then, the mapping $\mathbb{1}_{[0, t] \times A_{1}} \mapsto W^{H}\left([0, t] \times A_{1}\right)$ is an isometry between $\mathcal{S}$ and the linear space generated by $W^{H}$, and moreover, the mapping can be extended to $\mathscr{H}$. This isometry is denoted by

$$
\begin{aligned}
& \phi \longmapsto W^{H}(\phi)=\int_{0}^{T} \int_{\mathbb{R}} \phi(s, y) W^{H}(d s, d y), \\
& \phi \in \mathscr{H},
\end{aligned}
$$

and is called the Wiener integral with respect to $W^{H}$. Define the kernel $K_{H}(t, s)$ by

$$
\begin{aligned}
& K_{H}(t, s)=c_{H}\left(\left(\frac{t}{s}\right)^{H-1 / 2}(t-s)^{H-1 / 2}\right. \\
& \left.\quad+\left(\frac{1}{2}-H\right) s^{1 / 2-H} \int_{s}^{t} r^{H-3 / 2}(r-s)^{H-1 / 2} d r\right)
\end{aligned}
$$


for $H \in(0,1)$, where $c_{H}>0$ is a normalization constant given as follows:

$$
c_{H}=\sqrt{\frac{2 H \Gamma(3 / 2-H)}{\Gamma H+(1 / 2) \Gamma(2-2 H)}} .
$$

Consider a linear operator $\mathscr{K}_{H}^{*}: \mathcal{S} \mapsto L^{2}([0, T] \times \mathbb{R})$ defined by

$$
\begin{aligned}
& \left(\mathscr{K}_{H}^{*} \psi\right)(s, x) \\
& =K_{H}(T, s) \psi(s, x) \\
& \quad+\int_{s}^{T}(\psi(t, x)-\psi(s, x)) \frac{\partial K_{H}}{\partial t}(t, s) d t .
\end{aligned}
$$

Then, the operator $\mathscr{K}_{H}^{*}$ gives an isometry from $\mathscr{H}$ to $L^{2}([0, T] \times \mathbb{R})$, and we find that (see, for example, Nualart [20] and Tindel et al. [7]) the process

$$
W\left(A_{1} \times[0, t]\right):=W^{H}\left(\left(\mathscr{K}_{H}^{*}\right)^{-1}\left(1_{[0, t] \times A_{1}}\right)\right)
$$

defines a space-time white noise. Moreover, one can show that

$$
W^{H}(A \times[0, t])=\int_{0}^{t} \int_{A} K_{H}(t, s) W(d y, d s)
$$

for $t \in[0, T], A \in \mathscr{B}_{b}(\mathbb{R})$. In particular, when $H>1 / 2$ the kernel $K_{H}$ can be rewritten as

$$
\begin{aligned}
& K_{H}(t, s) \\
& \quad=\left(H-\frac{1}{2}\right) c_{H} s^{1 / 2-H} \int_{s}^{t} u^{H-1 / 2}(u-s)^{H-3 / 2} d u, \\
& \left(\mathscr{K}_{H}^{*} \psi(\cdot, x)\right)(t)=\int_{t}^{T} \frac{\partial K_{H}}{\partial t}(s, t) \psi(s, x) d s .
\end{aligned}
$$

The following result follows from Mémin et al. [25].

Proposition 5. For $H>1 / 2$ one has

$$
L^{1 / H}([0, T] \times \mathbb{R}) \subset \mathscr{H} .
$$

\section{Existence and Uniqueness of the Solution}

Let a filtered complete probability space $\left(\Omega, \mathscr{F},\left(\mathscr{F}_{t}\right)_{t \geq 0}, P\right)$ be given as in the previous section. In this section, we will study the existence and uniqueness of the solution to the stochastic equation

$$
\begin{aligned}
\frac{\partial u(t, x)}{\partial t}= & \left(\Delta+\Delta_{\alpha}\right) u(t, x)+\frac{\partial f}{\partial x}(t, x, u(t, x)) \\
& +\sigma(t, x, u(t, x)) \dot{L}+\dot{W}^{H},
\end{aligned}
$$

$t \geq 0, x \in \mathbb{R}$

$$
u(0, x)=u_{0}(x), \quad x \in \mathbb{R}
$$

where $\Delta$ is Laplacian, $\Delta_{\alpha}=-(-\Delta)^{\alpha / 2}$ is the fractional Laplacian generator on $\mathbb{R}, \dot{W}^{H}$ is the fractional noise, and $\dot{L}$ is a (pure jump) Lévy space-time white noise. Moreover, we assume also that Assumptions 1, 2, and 4 in Sections 1 and 2 hold.

From Walsh [26], one can introduce a notation of Walshmild solution to (35) by using the heat kernel $G^{(\alpha)}(s, y ; t, x)=$ $G^{(\alpha)}(t-s ; x-y)$ of $\Delta+\Delta_{\alpha}$. An $L^{p}(\Omega) \mathscr{F}_{t}$-adapted process $u:[0, T] \times \mathbb{R} \times \Omega \rightarrow \mathbb{R}$ is a solution to (35) if

$$
\begin{aligned}
& u(t, x)=\int_{\mathbb{R}} G^{(\alpha)}(t, x-y) u_{0}(y) d y \\
& +\int_{0}^{t} \int_{\mathbb{R}} G^{(\alpha)}(t-s, x-y) W^{H}(d s, d y) \\
& +\int_{0}^{t} \int_{\mathbb{R}} \frac{\partial G^{(\alpha)}}{\partial y}(t-s, x-y) f(s, y, u(s, y)) d y d s \\
& +\int_{0}^{t} \int_{\mathbb{R}} G^{(\alpha)}(t-s, x-y) \sigma(s, y, u(s, y)) \\
& \quad+(s, y) d y d s \\
& +\int_{0}^{t+} \int_{\mathbb{R} \times U_{2}} G^{(\alpha)}(t-s, x-y) \sigma(s, y, u(s, y)) \\
& \quad \cdot g(s, y, z) Q(d y, d z, d s) .
\end{aligned}
$$

In order to show the main theorem, we need the following lemma.

Lemma 6. Let $1 \leq r<+\infty$ and $1 \leq p^{\prime} \leq p<+\infty$ such that

$$
\frac{1}{r}=1+\frac{1}{p}-\frac{1}{p^{\prime}} \text {. }
$$

Define the operator 0 by

$$
\mathcal{O}(v)(t, x)=\int_{0}^{t} \int_{\mathbb{R}} \mathscr{G}(t-s, x-y) v(s, y) d y d s,
$$

for $v \in L^{1}\left([0, T], L^{p^{\prime}}(\mathbb{R})\right)$ and $\mathscr{G} \in\left\{G^{(\alpha)},\left(G^{(\alpha)}\right)^{2},(\partial / \partial y) G^{(\alpha)}\right\}$. Then, for all $t \in[0, T], \mathcal{O}$ is a bounded linear operator from $L^{1}\left([0, T], L^{p^{\prime}}(\mathbb{R})\right)$ to $L^{\infty}\left([0, T], L^{p}(\mathbb{R})\right)$. Specifically, we have the following:

(1) when $\mathscr{G}=G^{(\alpha)}$, we have

$$
\begin{aligned}
\| \mathcal{O} & (v)(t, \cdot) \|_{p} \\
& =\left\|\int_{0}^{t} \int_{\mathbb{R}} G^{(\alpha)}(t-s, \cdot-y) v(s, y) d y d s\right\|_{p} \\
& \leq C \int_{0}^{t}(t-s)^{(1-r) / 2 r}\|v(s, \cdot)\|_{p^{\prime}} d s ;
\end{aligned}
$$

(2) when $\mathscr{G}=\left(G^{(\alpha)}\right)^{2}$, we have

$$
\begin{aligned}
& \|\mathcal{O}(v)(t, \cdot)\|_{p} \\
& \quad=\left\|\int_{0}^{t} \int_{\mathbb{R}}\left(G^{(\alpha)}(t-s, \cdot-y)\right)^{2} v(s, y) d y d s\right\|_{p} \\
& \quad \leq C \int_{0}^{t}(t-s)^{(1-2 r) / 2 r}\|v(s, \cdot)\|_{p^{\prime}} d s ;
\end{aligned}
$$


(3) when $\mathscr{G}=(\partial / \partial y) G^{(\alpha)}$ and $r=1$, we have

$$
\begin{aligned}
& \|\mathcal{O}(v)(t, \cdot)\|_{p} \\
& \quad=\left\|\int_{0}^{t} \int_{\mathbb{R}} \frac{\partial G^{(\alpha)}}{\partial y}(t-s, \cdot-y) v(s, y) d y d s\right\|_{p} \\
& \quad \leq C \int_{0}^{t}(t-s)^{-1 / 2}\|v(s, \cdot)\|_{p} d s .
\end{aligned}
$$

Proof. Clearly, we have

$$
\begin{aligned}
& \int_{\mathbb{R}}\left((t-s)^{-1 / 2} e^{-|y|^{2} / C_{2}(t-s)}\right)^{r} d y \\
& \quad=C r^{-1 / 2}(t-s)^{(1-r) / 2}, \\
& \int_{\mathbb{R}}\left((t-s)^{-1 / 2} \wedge \frac{(t-s)}{|y|^{1+\alpha}}\right)^{r} d y \leq C(t-s)^{(1-r) / 2}
\end{aligned}
$$

for all $t>s>0$ and $1<\alpha<2$. It follows that

$$
\begin{aligned}
& \left\|G^{(\alpha)}(t-s, \cdot)\right\|_{r}=\left(\int_{\mathbb{R}}\left(G^{(\alpha)}(t-s, y)\right)^{r} d y\right)^{1 / r} \\
& \leq C\left(\int _ { \mathbb { R } } \left((t-s)^{-1 / 2} e^{-|y|^{2} / C_{2}(t-s)}+(t-s)^{-1 / 2}\right.\right. \\
& \left.\left.\wedge \frac{(t-s)}{|y|^{1+\alpha}}\right)^{r} d y\right)^{1 / r} \leq C(t-s)^{(1-r) / 2 r}
\end{aligned}
$$

for all $t>s>0$ and $1<\alpha<2$. Combining this with Minkowski's inequality, (9), and Young's inequality, we see that

$$
\begin{aligned}
& \|\mathcal{O}(v)(t, \cdot)\|_{p} \\
& \quad=\left\|\int_{0}^{t} \int_{\mathbb{R}} G^{(\alpha)}(t-s, \cdot-y) v(s, y) d y d s\right\|_{p} \\
& \leq C \int_{0}^{t}\left\|\int_{\mathbb{R}} G^{(\alpha)}(t-s, \cdot-y) v(s, y) d y\right\|_{p} d s \\
& \leq C \int_{0}^{t}\left\|\left(G^{(\alpha)}(t-s, \cdot) *|v(s, \cdot)|\right)(\cdot)\right\|_{p} d s \\
& \leq C \int_{0}^{t}\left\|G^{(\alpha)}(t-s, \cdot)\right\|_{r}\|v(s, \cdot)\|_{p^{\prime}} d s \\
& \leq C \int_{0}^{t}(t-s)^{(1-r) / 2 r}\|v(s, \cdot)\|_{p^{\prime}} d s,
\end{aligned}
$$

which gives case (1), and similarly, we can obtain case (2). Let us consider case (3).
For $t>s>0$, we denote $D_{1}=\{y \in \mathbb{R}|| y \mid<(t-$ $\left.s)^{3 / 2(1+\alpha)}\right\}$ and $\bar{D}_{1}$ denotes the complement of $D_{1}$. We then see that

$$
\begin{aligned}
& \left\|\frac{\partial G^{(\alpha)}}{\partial \cdot}(t-s, \cdot)\right\|_{1}=\int_{\mathbb{R}}\left|\frac{\partial G^{(\alpha)}}{\partial y}(t-s, y)\right| d y \\
& \quad \leq C \int_{D_{1}} \mid(t-s)^{1 / \alpha}\left((t-s)^{-1 / 2} e^{-|y|^{2} / C_{2}(t-s)}\right. \\
& \left.\quad+(t-s)^{-1 / 2}\right) \mid d y \\
& \quad+C \int_{\bar{D}_{1}} \mid \frac{1}{|y|}\left((t-s)^{-1 / 2} e^{-|y|^{2} / C_{2}(t-s)}\right. \\
& \left.\quad+\frac{t-s}{|y|^{1+\alpha}}\right) \mid d y \leq C(t-s)^{1 / \alpha-1 / 2} \\
& \quad \cdot \int_{D_{1}}\left|e^{-|y|^{2} / C_{2}(t-s)}+1\right| d y+C(t-s)^{-1 / 2} \\
& \quad \cdot \int_{\bar{D}_{1}}\left|\frac{1}{|y|} e^{-|y|^{2} / C_{2}(t-s)}+\frac{(t-s)^{3 / 2}}{|y|^{2+\alpha}}\right| d y \leq C(t \\
& \quad-s)^{-1 / 2}
\end{aligned}
$$

for $t>s>0$. It follows that

$$
\begin{aligned}
\|\mathcal{O}(v)(t, \cdot)\|_{p} \\
\quad=\left\|\int_{0}^{t} \int_{\mathbb{R}} \frac{\partial G^{(\alpha)}}{\partial y}(t-s, \cdot-y) v(s, y) d y d s\right\|_{p} \\
\leq C \int_{0}^{t}\left\|\left(\frac{\partial G^{(\alpha)}}{\partial \cdot}(t-s, \cdot) *|v(s, \cdot)|\right)(\cdot)\right\|_{p} d s \\
\leq C \int_{0}^{t}\left\|\frac{\partial G^{(\alpha)}}{\partial \cdot}(t-s, \cdot)\right\|_{1}\|v(s, \cdot)\|_{p} d s \\
\leq C \int_{0}^{t}(t-s)^{-1 / 2}\|v(s, \cdot)\|_{p} d s .
\end{aligned}
$$

This proves case (3) and the lemma follows.

Let $\mathscr{B}$ be the space of all $L^{p}(\mathbb{R})$-valued $\mathscr{F}_{t}$-adapted processes $u(t, \cdot)_{0 \leq t \leq T}:[0, T] \times \mathbb{R} \times \Omega \rightarrow \mathbb{R}$. For fixed $\eta>0$, define a functional $\|\cdot\|_{\mathscr{B}}$ on $\mathscr{B}$ by

$$
\|u\|_{\mathscr{B}}:=\left(\sup _{t \in[0, T]} e^{-\eta t} \mathbb{E}\left(\|u(t, \cdot)\|_{p}^{p}\right)\right)^{1 / p}
$$

for $u \in \mathscr{B}$. Then, $\|\cdot\|_{\mathscr{B}}$ is a norm on $\mathscr{B}$ and $\left(\mathscr{B},\|\cdot\|_{\mathscr{B}}\right)$ forms a Banach space. Consider the next integrals:

$$
\begin{gathered}
B_{u}^{0}(t, x):=\int_{\mathbb{R}} G^{(\alpha)}(t, x-y) u_{0}(y) d y, \\
B_{u}^{1}(t, x):=\int_{0}^{t} \int_{\mathbb{R}} \frac{\partial G^{(\alpha)}}{\partial y}(t-s, x-y) \\
\cdot f(s, y, u(s, y)) d y d s,
\end{gathered}
$$




$$
\begin{aligned}
& B_{u}^{2}(t, x):=\int_{0}^{t} \int_{\mathbb{R}} G^{(\alpha)}(t-s, x-y) W^{H}(d y, d s), \\
& B_{u}^{3}(t, x):=\int_{0}^{t} \int_{\mathbb{R}} G^{(\alpha)}(t-s, x-y) \sigma(s, y, u(s, y)) \\
& \cdot \psi(s, y) d y d s, \\
& B_{u}^{4}(t, x):=\int_{0}^{t} \int_{\mathbb{R} \times U_{2}} G^{(\alpha)}(t-s, x-y) \\
& \cdot \sigma(s, y, u(s, y)) g(s, y, z) Q(d z, d y, d s),
\end{aligned}
$$

for $u \in \mathscr{B}$ and $(t, x) \in[0, T] \times \mathbb{R}$ and define the operator

$$
\mathscr{B}(u)(t, x):=\sum_{i=0}^{4} B_{u}^{i}(t, x), \quad(t, x) \in[0, T] \times \mathbb{R}
$$

with $u \in \mathscr{B}$.

In this section, our main object is to expound and to prove the next theorem.

Theorem 7. Let $1 / 2<H<1$. Then, under Assumptions 1,2 , and 4, (35) admits a unique Walsh-mild solution $u=$ $\{u(t, x),(t, x) \in[0, T] \times \mathbb{R}\}$ such that

$$
\sup _{t \in[0, T]} \mathbb{E}\left[\|u(t, \cdot)\|_{p}^{p}\right]<\infty
$$

for all $T>0, \alpha \in(1,2)$, and $p \geq 4$.

Based on the fixed point principle on the set $\{u \in \mathscr{B}$ : $\left.u(0)=u_{0}\right\}$, in order to prove the theorem, it is enough to prove the following two statements:

(1) under Assumptions 1, 2, and $4, B_{u}^{i} \in \mathscr{B}$ for $i=0,1$, 2, 3, 4 and $u \in \mathscr{B}$;

(2) under Assumptions 1, 2, and 4 , the operator $\mathscr{B}(u)$ is a contraction on $\mathscr{B}$. In other words, there exists a constant $c \in(0,1)$ such that

$$
\|\mathscr{B}(u)-\mathscr{B}(v)\|_{\mathscr{B}} \leq c\|u-v\|_{\mathscr{B}}
$$

for $u, v \in \mathscr{B}$.

Proof of Statement (1). Given $\eta>0$, from (9), (46), Assumption 2, and Young's inequality for $1 / p=1+1 / p-1$, we have

$$
\begin{aligned}
\left\|B_{u}^{0}(t, x)\right\|_{p} & =\left\|\int_{\mathbb{R}} G^{(\alpha)}(t, x-y) u_{0}(y) d y\right\|_{p} \\
& \leq C\left\|\left(G^{(\alpha)}(t, \cdot) * u_{0}(\cdot)\right)(\cdot)\right\|_{p} \\
& \leq C\left\|G^{(\alpha)}(t, \cdot)\right\|_{1} \cdot\left\|u_{0}(\cdot)\right\|_{p} \leq C\left\|u_{0}(\cdot)\right\|_{p} \\
& <\infty .
\end{aligned}
$$

Consider $B_{u}^{1}$ and take $r=1$. It follows from Lemma 6 and Assumption 1 that

$$
\begin{aligned}
& \mathbb{E}\left\|B_{u}^{1}(t, x)\right\|_{p}^{p}=\mathbb{E} \| \int_{0}^{t} \int_{\mathbb{R}} \frac{\partial G^{(\alpha)}}{\partial y}(t-s, x-y) \\
& \cdot f(s, y, u(s, y)) d y d s \|_{p}^{p} \\
& \leq C \mathbb{E}\left(\int_{0}^{t}\left\|\frac{\partial G^{(\alpha)}}{\partial \cdot}(t-s, \cdot)\right\|_{1} \cdot \| f(s,\right. \\
& \left.\cdot, u(s, \cdot)) \|_{p} d s\right)^{p} \leq C \mathbb{E}\left(\int_{0}^{t}(1\right. \\
& \left.\left.+\|u(s, \cdot)\|_{p}\right) d s\right)^{p} \leq C(1 \\
& \left.+\sup _{s \in[0, T]} \mathbb{E}\|u(s, \cdot)\|_{p}^{p}\right) \leq C\left(1+\|u(s, \cdot)\|_{\mathscr{B}}^{P}\right)<\infty
\end{aligned}
$$

for all $u \in \mathscr{B}$, which gives $B_{u}^{1}(t, x) \in \mathscr{B}$.

For $B_{u}^{2}$, by Proposition 5 , we deduce that

$$
\begin{aligned}
& \mathbb{E}\left\|B_{u}^{2}(t, x)\right\|_{p}^{p}=\mathbb{E}\left\|\int_{0}^{t} \int_{\mathbb{R}} G^{(\alpha)}(t-s, x-y) W^{H}(d y, d s)\right\|_{p}^{p} \\
& =\int_{\mathbb{R}} \mathbb{E}\left|\int_{0}^{t} \int_{\mathbb{R}} G^{(\alpha)}(t-s, x-y) W^{H}(d y, d s)\right|^{p} d x \\
& =\int_{\mathbb{R}} \mathbb{E}\left|\int_{0}^{t} \int_{\mathbb{R}}\left(K_{H}^{*} G^{(\alpha)}(t-\cdot, x-\cdot)\right)(s, y) W(d y, d s)\right|^{p} d x \\
& \leq C \int_{\mathbb{R}}\left\langle K_{H}^{*} G^{(\alpha)}(t-\cdot, x-\cdot), K_{H}^{*} G^{(\alpha)}(t-\cdot, x-\cdot)\right\rangle_{L^{2}([0, T] \times \mathbb{R})}^{p / 2} d x \\
& =C \int_{\mathbb{R}}\left\langle G^{(\alpha)}(t-\cdot, x-\cdot), G^{(\alpha)}(t-\cdot, x-\cdot)\right\rangle_{\mathscr{H}}^{p / 2} d x \\
& \leq C \int_{\mathbb{R}}\left\|G^{(\alpha)}(t-\cdot, x-\cdot)\right\|_{L^{1 / H}([0, T] \times \mathbb{R})}^{p} d x .
\end{aligned}
$$

Moreover, similar to the proof of Lemma 6, we have

$$
\begin{aligned}
& \left\|G^{(\alpha)}(t-\cdot, x-\cdot)\right\|_{L^{1 / H}([0, T] \times \mathbb{R})}^{p} \\
& \quad=\left(\int_{0}^{t} \int_{\mathbb{R}}\left|G^{(\alpha)}(t-s, x-y)\right|^{1 / H} d y d s\right)^{p H} \\
& \quad \leq C\left(\int_{0}^{t}(t-s)^{(H-1) / 2 H} d s\right)^{p H}<\infty .
\end{aligned}
$$

This gives $B_{u}^{2}(t, x) \in \mathscr{B}$. 
For $B_{u}^{3}$, by Lemma 6 with $1 / r=1-2 / p+1 / p=1-1 / p$ and Assumption 4, it follows that

$$
\begin{aligned}
& \mathbb{E}\left\|B_{u}^{3}(t, x)\right\|_{p}^{p}=\mathbb{E} \| \int_{0}^{t} \int_{\mathbb{R}} G^{(\alpha)}(t-s, x-y) \\
& \cdot \sigma(s, y, u(s, y)) \psi(s, y) d y d s \|_{p}^{p} \\
& \leq C \mathbb{E}\left(\int_{0}^{t}(t-s)^{(1-r) / 2 r}\|1+\mid u(s, \cdot)\|_{p}\right. \\
& \left.\cdot\|\psi(t, \cdot)\|_{p} d s\right)^{p} \leq C\left(\int_{0}^{t}(t-s)^{(1-r) / 2 r} d s\right)^{p}(1 \\
& \left.+\|u(\cdot)\|_{\mathscr{B}}^{p}\right) \sup _{t \in[0, T]}\|\psi(t, \cdot)\|_{p}^{p} \leq C \sup _{t \in[0, T]}\|\psi(t, \cdot)\|_{p}^{p} \\
& \cdot\left(1+\|u(\cdot)\|_{\mathscr{B}}^{p}\right) .
\end{aligned}
$$

Finally, let us estimate $B_{u}^{4}(t, x)$. From Assumption 4 , Lemma 6 with $1 / r=2 / p-4 / p+1=1-2 / p \in(0,1]$, and Proposition 3 , it follows that

$$
\begin{aligned}
& \mathbb{E}\left\|B_{u}^{4}(t, x)\right\|_{p}^{p} \leq C \int_{\mathbb{R}}\left(\int_{0}^{t} \int_{\mathbb{R} \times U_{2}} v_{2}(d z) d y d s\right. \\
& \left.\cdot\left(\mathbb{E}\left|G^{(\alpha)}(t-s, x-y) \sigma(s, y, u(s, y)) g(s, y, z)\right|^{p}\right)^{2 / p}\right)^{p / 2} \\
& \leq C \int_{\mathbb{R}}\left(\int_{0}^{t} \int_{\mathbb{R} \times U_{2}}\left|G^{(\alpha)}(t-s, x-y) g(s, y, z)\right|^{2}\right. \\
& \left.\cdot\left(\mathbb{E}\left(1+|u(s, y)|^{p}\right)\right)^{2 / p} v_{2}(d z) d y d s\right)^{2 / p} d x \\
& =C \| \int_{0}^{t} \int_{\mathbb{R}}\left|G^{(\alpha)}(t-s, \cdot-y)\right|^{2} \cdot\left(\int_{U_{2}}|g(s, \cdot z)|^{2} v_{2}(d z)\right) \\
& \cdot\left(1+\mathbb{E}|u(s, y)|^{p}\right)^{2 / p} d y d s \|_{p / 2}^{p / 2} \\
& \leq C\left(\int_{0}^{t}\left\|\left(G^{(\alpha)}(t-s, \cdot)\right)^{2}\right\|_{r}\right. \\
& \left.\cdot\left\|\int_{U_{2}}|g(s, \cdot, z)|^{2} v_{2}(d z)\left(1+\mathbb{E}|u(s, \cdot)|^{p}\right)^{2 / p}\right\|_{p / 4} d s\right)^{p / 2} \\
& \leq C\left(\int_{0}^{t}(t-s)^{(1-2 r) / 2 r} d s\right)^{p / 2} \cdot \sup _{t \in[0, T]}\left\|\left(1+\mathbb{E}|u(s, \cdot)|^{p}\right)^{2 / p}\right\|_{p / 2}^{p / 2} \\
& \cdot \sup _{t \in[0, T]}\left\|\int_{U_{2}}|g(s, \cdot, z)|^{2} \nu_{2}(d z)\right\|_{p / 2}^{p / 2} \leq C\left(1+\|u(\cdot)\|_{\mathscr{B}}^{p}\right) \\
& \sup _{t \in[0, T]}\left\|\int_{U_{2}}|g(s, \cdot, z)|^{2} \nu_{2}(d z)\right\|_{p / 2}^{p / 2}<\infty .
\end{aligned}
$$

Thus, we have showed that the operators $B_{u}^{i}, i=0,1,2,3,4$ defined by (48) map $\mathscr{B}$ to itself. On the other hand, in some same ways as in estimates (52)-(57), one can show that $B_{u}^{i} \epsilon$ $\mathscr{B}$ when $\eta>0$ sufficiently large. This completes the proof.

Proof of Statement (2). Suppose $u_{0}$ and $v_{0}$ are initials of $\left(\mathscr{F}_{t}\right)_{t \geq 0}$-adapted random fields $u, v \in \mathscr{B}$ such that $u_{0}=v_{0}$. We start with estimating $B_{u}^{1}$. Note that

$$
\begin{aligned}
& \mathbb{E}\left(\left\|B_{u}^{1}(t, \cdot)-B_{v}^{1}(t, \cdot)\right\|_{p}^{p}\right) \\
& =\mathbb{E} \| \int_{0}^{t} \int_{\mathbb{R}} \frac{\partial G^{(\alpha)}}{\partial y}(t-s, \cdot-y) \\
& \cdot|f(s, y, u(s, y))-f(s, y, v(s, y))| d y d s \|_{p}^{p} \\
& \leq C \mathbb{E}\left(\int_{0}^{t}(t-s)^{(1-r) / 2 r} \| f(s, y, u(s, y))\right. \\
& \left.-f(s, y, v(s, y)) \|_{p} d s\right)^{p} \leq C \mathbb{E}\left(\int_{0}^{t}(t\right. \\
& \left.-s)^{(1-r) / 2 r} \mathbb{E}\|u(s, \cdot)-v(s, \cdot)\|_{p} d s\right)^{p}
\end{aligned}
$$

for $p^{\prime}=p / 3$ by (3) and Lemma 6 with $1 / r=1 / p-1 / p^{\prime}+1=$ $1-2 / p$. We get that

$$
\begin{aligned}
& \left\|B_{u}^{1}(t, \cdot)-B_{v}^{1}(t, \cdot)\right\|_{\mathscr{B}}^{p}=\sup _{t \in[0, T]} e^{-\eta t} \mathbb{E}\left(\| B_{u}^{1}(t, \cdot)\right. \\
& \left.-B_{v}^{1}(t, \cdot) \|_{p}^{p}\right) \leq C \sup _{t \in[0, T]} \mathbb{E}\left(\int_{0}^{t}(t-s)^{(1-r) / 2 r}\right. \\
& \left.\cdot e^{-\eta t / p}\|u(s, \cdot)-v(s, \cdot)\|_{p} d s\right)^{p} \\
& \leq C \sup _{t \in[0, T]} \mathbb{E}\left(\int_{0}^{t} e^{-\eta s}\|u(s, \cdot)-v(s, \cdot)\|_{p}^{p} d s\right) \\
& \cdot\left(\int_{0}^{t}\left((t-s)^{(1-r) / 2 r} e^{-\eta(t-s) / p}\right)^{p /(p-1)} d s\right)^{p-1} \\
& \leq C \sup _{t \in[0, T]} \int_{0}^{t} e^{-\eta s} \mathbb{E}\|u(s, \cdot)-v(s, \cdot)\|_{p}^{p} d s \\
& \cdot\left(\int_{0}^{t}\left((t-s)^{(1-r) / 2 r} e^{-\eta(t-s) / p}\right)^{p /(p-1)} d s\right)^{p-1} \\
& \leq C\|u-v\|_{\mathscr{B}}^{p} \\
& \cdot\left(\int_{0}^{\infty}\left((t-s)^{(1-r) / 2 r} e^{-\eta(t-s) / p}\right)^{p /(p-1)} d s\right)^{p-1} \\
& \quad=C\|u-v\|_{\mathscr{B}}^{p}\left(\frac{p-1}{\eta}\right)^{(\kappa+1)(p-1)}\left(\int_{0}^{\infty} e^{-x} x^{\kappa} d x\right)^{p-1} \\
& \leq C T\|u-v\|_{\mathscr{B}}^{p}\left(\frac{p-1}{\eta}\right)^{(\kappa+1)(p-1)}(\Gamma(\kappa+1))^{p-1},
\end{aligned}
$$


where $\kappa=p(1-r) / 2 r(1-p)$, which implies that

$$
\begin{aligned}
& \left\|B_{u}^{1}(t, \cdot)-B_{v}^{1}(t, \cdot)\right\|_{\mathscr{B}} \\
& \leq C\left(\frac{p-1}{\eta}\right)^{(\kappa+1)((p-1) / p)}(\Gamma(\kappa+1))^{(p-1) / p} \\
& \cdot\|u-v\|_{\mathscr{B}} \leq c\|u-v\|_{\mathscr{B}}
\end{aligned}
$$

with $c \in(0,1)$ by choosing $\eta>0$ large enough.

Next we consider $B_{u}^{3}$. We have that

$$
\begin{aligned}
\mathbb{E}\left\|B_{u}^{3}(t, \cdot)-B_{v}^{3}(t, \cdot)\right\|_{p}^{p}=\mathbb{E} \| \int_{0}^{t} \int_{\mathbb{R}} G^{(\alpha)}(t-s, \cdot-y) \\
\cdot \psi(s, y) \\
\cdot|\sigma(s, y, u(s, y))-\sigma(s, y, v(s, y))| d y d s \|_{p}^{p}
\end{aligned}
$$

$$
\begin{aligned}
& \leq C \mathbb{E}\left(\int_{0}^{t}(t-s)^{(1-r) / 2 r}\|\psi(s, \cdot)\|_{p} \| \sigma(s, y, u(s, y))\right. \\
& \left.-\sigma(s, y, v(s, y)) \|_{p} d s\right)^{p} \leq C \sup _{t \in[0, T]}\|\psi(t, \cdot)\|_{p}^{p} \\
& \cdot \mathbb{E}\left(\int_{0}^{t}(t-s)^{(1-r) / 2 r} \| \sigma(s, y, u(s, y))\right. \\
& \left.-\sigma(s, y, v(s, y)) \|_{p} d s\right)^{p} .
\end{aligned}
$$

Thus, a similar procedure as above implies that $B_{u}^{3}$ is a contraction on $\mathscr{B}$ by taking $\eta>0$ larger enough.

We finally consider $B_{u}^{4}$. By the generalized B-D-G inequality (21) in Proposition 3, similar to (57), we can see that

$$
\begin{aligned}
& \mathbb{E}\left(\left\|B_{u}^{4}(t, \cdot)-B_{v}^{4}(t, \cdot)\right\|_{\mathscr{B}}^{p}\right)=\sup _{t \in[0, T]} e^{-\eta t} \mathbb{E}\left(\left\|B_{u}^{4}(t, \cdot)-B_{v}^{4}(t, \cdot)\right\|_{p}^{p}\right) \\
& \quad=\sup _{t \in[0, T]} e^{-\eta t} \int_{\mathbb{R}} \mathbb{E}\left|\int_{0}^{t+} \int_{\mathbb{R} \times U_{2}} G^{(\alpha)}(t-s, x-y) g(s, y, z) \cdot(\sigma(s, y, u(s, y))-\sigma(s, y, v(s, y))) Q(d z, d y, d s)\right|^{p} d x \\
& \quad \leq c\|u-v\|_{\mathscr{B}}^{p},
\end{aligned}
$$

with $c \in(0,1)$ by choosing $\eta>0$ large enough.

To sum up, we have shown that $\mathscr{B}(u)$ is a contraction on $\mathscr{B}$ for $\eta>0$ large enough and statement (2) follows.

Remark 8. From the proof above, one can see that Theorem 7 is also true for $H=1 / 2$.

\section{Conflicts of Interest}

The authors declare that there are no conflicts of interest regarding the publication of this paper.

\section{Authors' Contributions}

Dengfeng Xia, Litan Yan, and Weiyin Fei carried out the mathematical studies, participated in the sequence alignment, drafted the manuscript, and participated in the design of the study and performed proof of results. All authors read and approved the final manuscript.

\section{Acknowledgments}

The work is supported and sponsored by National Natural Science Foundation of China (Grant nos. 11571071, 71271003, and 71571001), Natural Science Foundation of Anhui Province (Grant no. 1608085MA02), and the Foundation for Young Talents in College of Anhui Province (Grant no. gxyq2017014).

\section{References}

[1] T. E. Duncan, B. Pasik-Duncan, and B. Maslowski, "Fractional Brownian motion and stochastic equations in Hilbert spaces," Stochastics and Dynamics, vol. 2, no. 2, pp. 225-250, 2002.

[2] Y. Hu, "Heat equations with fractional white noise potentials," Applied Mathematics and Optimization, vol. 43, no. 3, pp. 221243, 2001.

[3] Y. Jiang, X. Wang, and Y. Wang, "On a stochastic heat equation with first order fractional noises and applications to finance," Journal of Mathematical Analysis and Applications, vol. 396, no. 2, pp. 656-669, 2012.

[4] Y. Jiang, T. Wei, and X. Zhou, "Stochastic generalized Burgers equations driven by fractional noises," Journal of Differential Equations, vol. 252, no. 2, pp. 1934-1961, 2012.

[5] J. Liu and L. Yan, "On a semilinear stochastic partial differential equation with double-parameter fractional noises," Science China. Mathematics, vol. 57, no. 4, pp. 855-872, 2014.

[6] K. Sobczyk, Stochastic Differential Equations with Applications to Physics and Engineering, vol. 40 of Mathematics and Its Applications (East European Series), Kluwer Academic, London, UK, 1991.

[7] S. Tindel, C. A. Tudor, and F. Viens, "Stochastic evolution equations with fractional Brownian motion," Probability Theory and Related Fields, vol. 127, no. 2, pp. 186-204, 2003.

[8] Y. Mishura, G. Shevchenko, and E. Valkeila, "Random variables as pathwise integrals with respect to fractional Brownian motion," Stochastic Processes and Their Applications, vol. 123, no. 6, pp. 2353-2369, 2013. 
[9] L. Bo, Y. Jiang, and Y. Wang, "On a class of stochastic Anderson models with fractional noises," Stochastic Analysis and Applications, vol. 26, no. 2, pp. 256-273, 2008.

[10] L. Bo and Y. Wang, "Stochastic Cahn-Hilliard partial differential equations with Lévy spacetime white noises," Stochastics and Dynamics, vol. 6, no. 2, pp. 229-244, 2006.

[11] K. Shi and Y. Wang, "On a stochastic fractional partial differential equation driven by a Lévy space-time white noise," Journal of Mathematical Analysis and Applications, vol. 364, no. 1, pp. 119-129, 2010.

[12] C. Mueller, “The heat equation with Lévy noise," Stochastic Processes and their Applications, vol. 74, no. 1, pp. 67-82, 1998.

[13] Z.-Q. Chen, P. Kim, and R. Song, "Global heat kernel estimate for relativistic stable processes in exterior open sets," Journal of Functional Analysis, vol. 263, no. 2, pp. 448-475, 2012.

[14] A. Løkka, B. Øksendal, and F. Proske, "Stochastic partial differential equations driven by Lévy space-time white noise," The Annals of Applied Probability, vol. 14, no. 3, pp. 1506-1528, 2004.

[15] A. Truman and J.-L. Wu, "Stochastic Burgers equation with Lévy space-time white noise," in Probabilistic Methods in Fluids, I. M. Davies, N. Jacob, A. Truman, O. Hassan, K. Morgan, and N. P. Weatherill, Eds., pp. 298-323, World Scientific, Singapore, 2003.

[16] A. Truman and J. L. Wu, "Fractal Burgers equation driven by Lévy noises," in SPDE and Applications, G. Da Prato and L. Tabaro, Eds., vol. 245 of Lecture Notes in Pure and Applied Mathematics, pp. 295-310, Chapman and Hall, CRC Taylor and Francis Group, Boca Raton, Fla, USA, 2006.

[17] D. Xia and L. Yan, "On a semilinear mixed fractional heat equation driven by fractional Brownian sheet," Boundary Value Problems, vol. 2017, article 7, 24 pages, 2017.

[18] Z.-Q. Chen, P. Kim, and R. Song, "Dirichlet heat kernel estimates for $\Delta^{\alpha / 2}+\Delta^{\beta / 2}$," Illinois Journal of Mathematics, vol. 54, no. 4, pp. 1357-1392, 2010.

[19] Z. Chen, P. Kim, and R. Song, "Heat kernel estimates for $\Delta+\Delta^{\alpha / 2}$ in $C^{1,1}$ open sets," Journal of the London Mathematical Society, vol. 84, pp. 58-80, 2011.

[20] D. Nualart, The Malliavin Calculus and Related Topics, Springer, Berlin, Germany, 2006.

[21] V. Kolokoltsov, "Symmetric stable laws and stable-like jumpdiffusions," Proceedings of the London Mathematical Society, vol. 80, no. 3, pp. 725-768, 2000.

[22] R. F. Bass and D. A. Levin, "Transition probabilities for symmetric jump processes," Transactions of the American Mathematical Society, vol. 354, no. 7, pp. 2933-2953, 2002.

[23] N. Ikeda and S. Watanabe, Stochastic Differential Equations and Diffusion Processes, North-Holland/Kodansha, New York, NY, USA, 1981.

[24] C. Knoche, "SPDEs in infinite dimensional with Poisson noise," Comptes Rendus Mathematique. Academie des Sciences. Paris, vol. 339, no. 9, pp. 647-652, 2004.

[25] J. Mémin, Y. Mishura, and E. Valkeila, "Inequalities for the moments of Wiener integrals with respect to a fractional Brownian motion," Statistics \& Probability Letters, vol. 51, no. 2, pp. 197-206, 2001.

[26] J. B. Walsh, "An introduction to stochastic partial differential equations," in Ecole d'été de Probabilités de Saint Flour XIV, vol. 1180 of Lecture Notes in Mathematics, pp. 266-439, Springer, Berlin, Germany, 1986. 


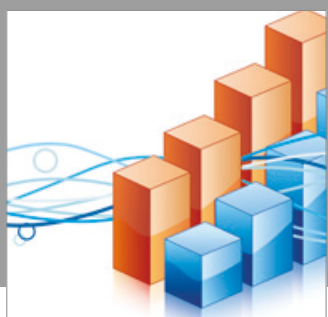

Advances in

Operations Research

vatersals

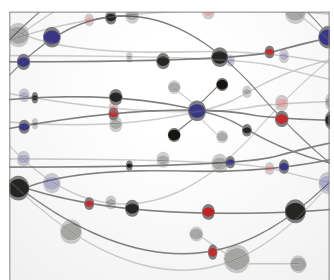

\section{The Scientific} World Journal
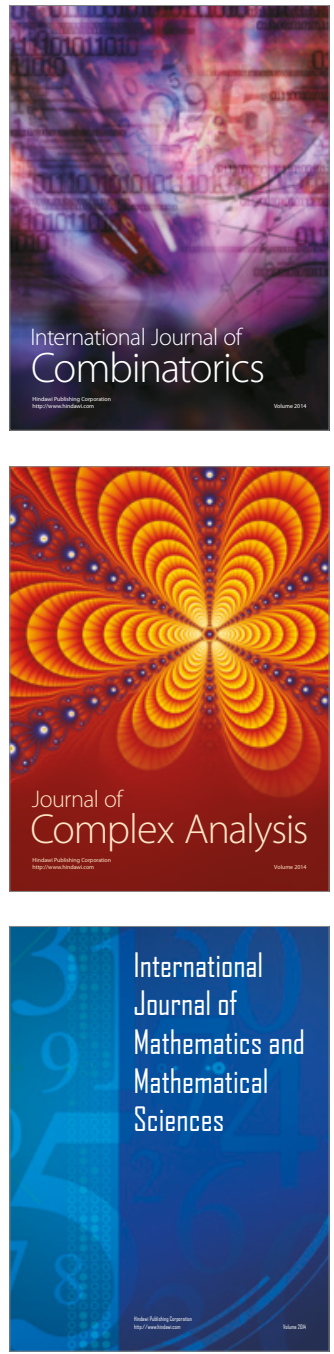
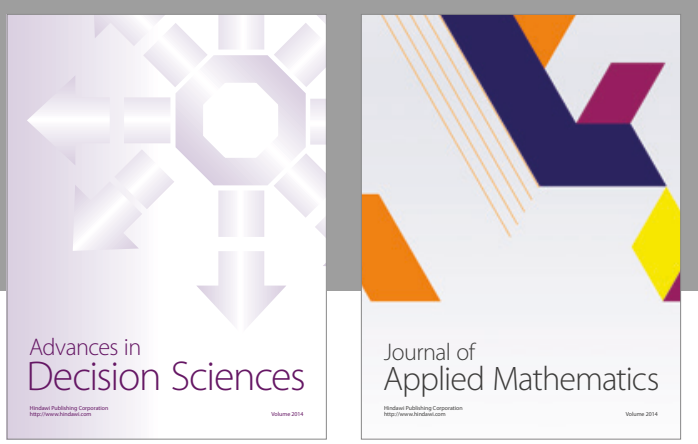

Algebra

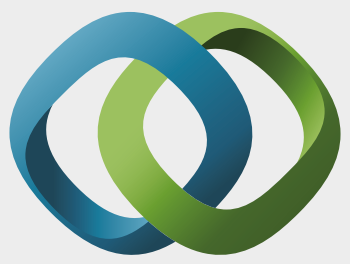

\section{Hindawi}

Submit your manuscripts at

https://www.hindawi.com
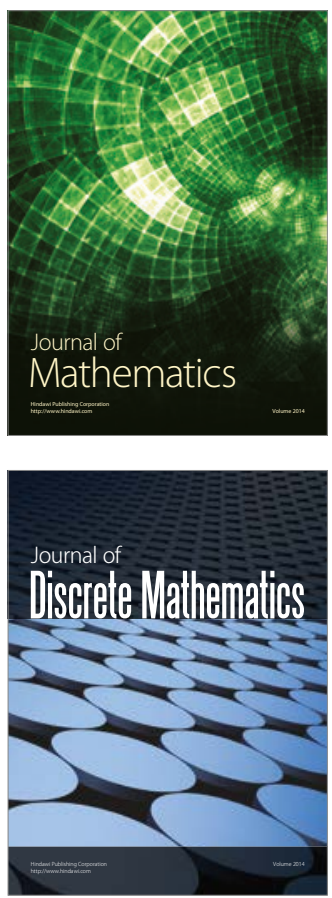

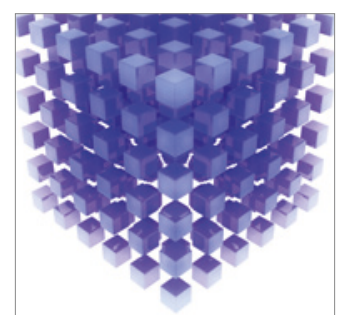

Mathematical Problems in Engineering
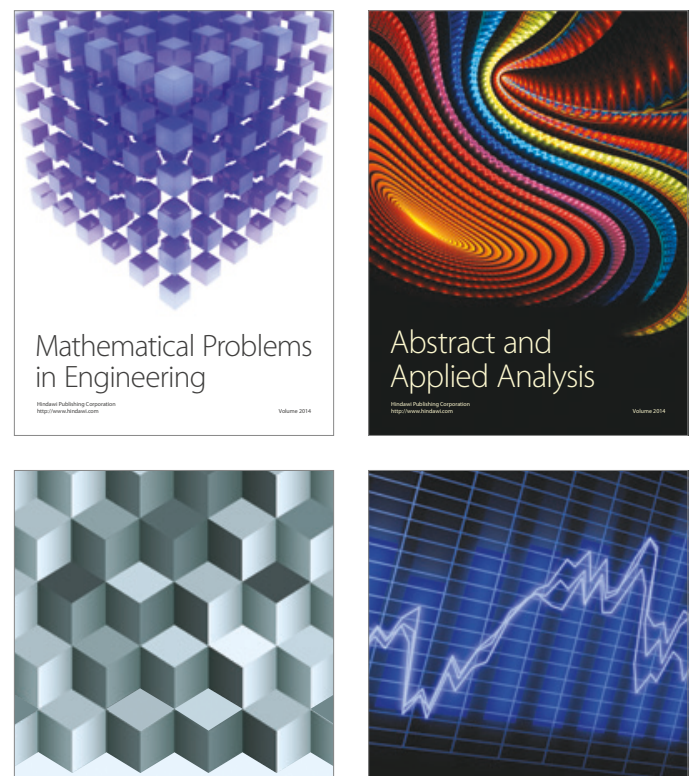

Journal of

Function Spaces

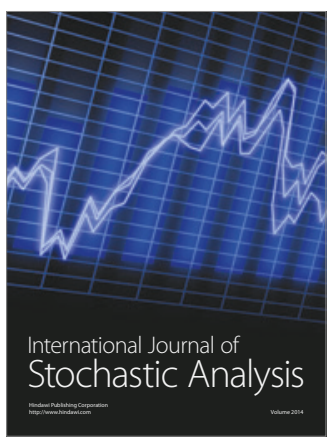

Probability and Statistics
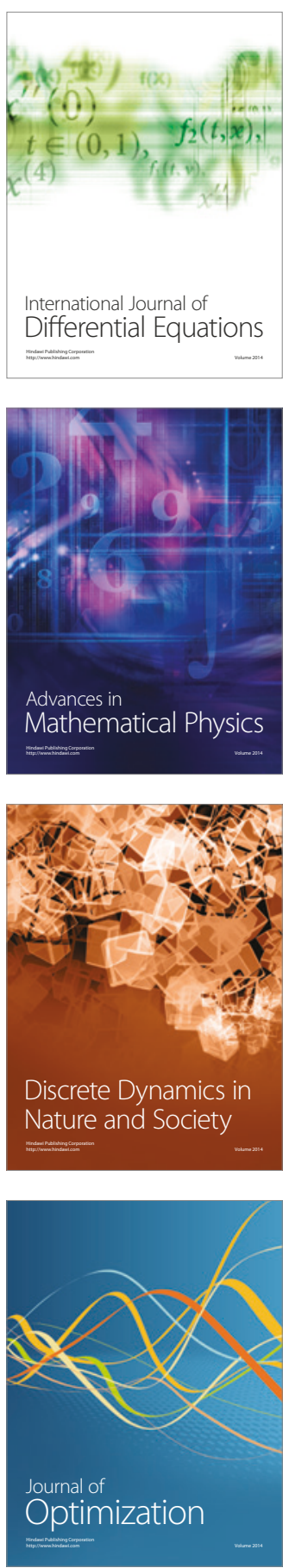